\title{
Size-dependent mechanical behavior and boundary layer effects in entangled metallic wire material systems
}

\author{
Yanhong $\mathrm{Ma}^{1,2}$, Qicheng Zhang ${ }^{1}$, Dayi Zhang ${ }^{1, *} \oplus$, Fabrizio Scarpa ${ }^{3}$, Di Gao ${ }^{1}$, and Jie Hong ${ }^{1,2}$ \\ ${ }^{1}$ School of Energy and Power Engineering, Beihang University, Beijing 100083, People's Republic of China \\ ${ }^{2}$ Collaborative Innovation Center of Advanced Aero-Engine, Beihang University, Beijing 100083, People's Republic of China \\ ${ }^{3}$ Advanced Composites Centre for Innovation and Science, University of Bristol, Bristol BS8 1TR, UK
}

Received: 1 July 2016

Accepted: 4 October 2016

Published online:

8 December 2016

(C) The Author(s) 2016. This article is published with open access at Springerlink.com

\begin{abstract}
This paper describes the influence on the compressive and dissipative behavior of entangled metallic wire material (EMWM) samples provided by their size and mutual connectivity. The mechanical properties of EMWM specimens with different thicknesses are obtained from quasi-static compressive and cyclic loading. The behavior of the stress-strain curves, tangent modulus, and loss factor are strongly dependent on the thickness of the samples. The analysis from samples connected in different layouts shows that apart from the global thickness, size scale effects and contact interface also play important roles in controlling the behavior of EMWM systems. The importance of the sample size and its connectivity with adjacent different layers are defined by the unique microstructure and contact properties of the wires near the specimen boundary layer. The boundary layer produces different mechanical behaviors and a distinct structural configuration compared to the EMWM bulk solid. These peculiar characteristics are confirmed by microstructural and qualitative observations obtained from computed tomography scanning. The results and analysis presented in this work are relevant to designing EMWM material systems with adaptive performance under different loading and geometric constraints.
\end{abstract}

\section{Introduction}

Entangled metallic wire material (EMWM) [1-3] (also called metal mesh [4-6] in earlier literature) is a form of porous material made from tangled metallic helix wires. Entangled metallic wire structures are manufactured via a process of wire drawing, weaving, and compression molding. The mechanical behavior of an EMWM solid is similar to the one of an elastomeric rubber; hence, the term 'metal rubber' sometimes is used to define EMWM [7-9]. The material is suitable for applications related to vibration alleviation and sound absorption in critical environmental conditions [10-12]. EMWM prototypes have also been developed as heat transfer insulators [13], active vibration control elements [8, 14], and even as surgical implants [15].

The static mechanical behavior of EMWM is mainly affected by the dimensions of the wire, its

Address correspondence to E-mail: dayi@buaa.edu.cn 
material properties, the weaving process used, and the relative density obtained from the production layout $[9,16]$. It must be noticed that rather than the relative density, the porosity is sometimes used as a defining parameter, because of the similarity between EMWM and porous materials [2, 3, 17]. Ma et al. [18] have studied the effects of the wire diameter, material, helix dimension, and relative density on the compressive properties of EMWM. Zhang et al. [16] have tested EMWM samples produced from nickelbased superalloys, and identified the relative density as one of the most important factors affecting the tangent modulus, Poisson's ratio, and damping of EMWM. Tan et al. [1] have investigated the uniaxial tensile behavior and mechanical properties of EMWM, and have also shown that the porosity strongly influences the performance of the material. Tan et al. [19] have also studied the mechanical behavior of quasi-ordered entangled aluminum alloy wire materials, and have found that this porous solid exhibits a three-stage stress-strain behavior under uniaxial compressive loading. Entangled titanium wire [20] and steel wire [6] solids also exhibit the three-stage stress-strain curves after heat treatment, and that is similar to what observed in other metal and polymeric porous materials [21]. Dynamic mechanical analysis on nickel wire-based EMWM solids indicates that the dynamic properties (storage modulus and loss factor) are characterized by the pulsation of the dynamic force and the dynamic deformation. The parameters of the dynamic excitation influence the equivalent mechanical properties in a way that is quite different from the one related to analogous mechanical performance under static loading [7].

Although the compressive mechanical properties of EMWM have been evaluated in a fairly comprehensive manner, an aspect that has been overlooked so far is the size-dependent behavior of EMWM samples. Size effects are well known to affect the failure load and behavior of composite materials [22-24], as well as the energy density in drilled cylindrical concrete specimens [25]. Unlike other metallic materials, the relative scale that exists between the representative volume element of the EMWM (i.e., the finite helix wires) and the EMWM solid is not negligible. Size effects can therefore be expected to occur during the mechanical loading of the samples, which implies that the dimension of an EMWM specimen can influence the properties of the material, such as the tangent modulus and the loss factor.

The objective of the present work is to understand from an experimental perspective how size and connectivity between different groups of entangled metal wires affect the mechanical compressive performance of EMWM systems. EMWM samples with different thicknesses have been produced and subjected to quasi-static compression loading. The results show that the sample thickness has a significant influence on the global mechanical compression properties. Moreover, combinations of EMWM samples disposed in parallel and in series with and without separation by rigid plates have also been investigated for practical design purposes. We observe some quite interesting and distinct mechanical behaviors occurring for the different combination methods, due to the existence of a finite region at the boundary (i.e., the boundary layer, BL). The effects of the BL on the global mechanical properties of EMWM systems are also discussed using a qualitative model.

\section{EMWM specimens}

The EMWM samples have been produced from austenitic stainless steel wires (alloy 0Cr18Ni9) with a diameter of $0.11 \mathrm{~mm}$ (ANPING DONGSHENG Hardware and Wiremesh CO., LTD, P. R. China). The nominal chemical composition and the mechanical properties of the wires are listed in Table 1.

The production of the EMWM samples was performed in the following process. The stainless steel
Table 1 Chemical compositions (wt $\%$ ) and mechanical properties of the wires

\begin{tabular}{llllllll}
\hline Fe & $\mathrm{Ni}$ & $\mathrm{C}$ & $\mathrm{Si}$ & $\mathrm{Mn}$ & $\mathrm{S}$ & $\mathrm{P}$ & $\mathrm{Cr}$ \\
\hline Balance & 8.01 & $\leq 0.080$ & $\leq 1.00$ & $\leq 2.00$ & $\leq 0.03$ & $\leq 0.035$ & 17.12 \\
\hline Young's modulus/GPa & \multicolumn{2}{c}{ Tensile strength/MPa } & \multicolumn{2}{c}{ Yield stress/MPa } & \multicolumn{2}{c}{ Elongation $/ \delta \%$} \\
\hline 199 & 680 & \multicolumn{2}{c}{340} & \multicolumn{2}{c}{54} \\
\hline
\end{tabular}


wire has been initially wound into a helix and then subjected to uniform tension (this process is called wire drawing). The stretched wire has been then weaved into a crisscross pattern to obtain a base porous material. The base samples have been then placed into a specially designed mold, and shaped into form by applying a compressive force ranging between 12 and $16 \mathrm{kN}$ for at least $30 \mathrm{~s}$. The compression loading is tailored to provide a specific relative density for the specimens. In our case, the relative density is defined as the ratio between the EMWM's and the wire's densities:

$\bar{\rho}=\frac{\rho_{\mathrm{EMWM}}}{\rho_{\mathrm{WIRE}}}=\frac{M / V}{\rho_{\mathrm{WIRE}}}$.

In Eq. (1), $\rho_{\mathrm{EMWM}}$ is calculated as the ratio between the mass $M$ and the volume $V$ of each sample. The wire density $\rho_{\text {WIRE }}$ is the density of the $0 \mathrm{Cr} 18 \mathrm{Ni} 9$ material. All the samples have been produced with a relative density close to 0.21 . The characteristics of the samples are listed in Tables 2 and 3, and the photographs of some specimens are shown in Fig. 1. Figure 1a illustrates five samples with equal base cross section, but different thicknesses corresponding to the molding direction (the blue arrow in Fig. 1) during the manufacturing. The direction also represents the one related to the compression loading. Another batch of samples with the same thickness but different base cross sections has been produced (Fig. 1b), and then connected in parallel (Fig. 1c). Figure 1d shows another type of connectivity used in the EMWM systems evaluated in this paper. The three entangled metal wire structures have a similar total thickness, but they are made with combinations of five, two, and single EMWM samples in series. Figure 1e shows five samples connected in series, each EMWM sample is however separated by thin steel plates whose thickness is $0.5 \mathrm{~mm}$ each.

\section{Experimental methods and data processing}

Quasi-static cyclic compression tests have been carried out to investigate the mechanical properties of each specimen, with particular focus on the tangent modulus and the loss factor during the hysteretic
Table 2 The parameters of the cuboid samples with different thicknesses

\begin{tabular}{lllllll}
\hline Set & Specimen name & Width/mm & Depth/mm & Thickness/mm & Mass/g & Relative density \\
\hline A1 & A1-1 & 20.8 & 20.8 & 2.3 & 1.58 & 0.202 \\
& A1-2 & 20.9 & 20.8 & 2.3 & 1.56 & 0.199 \\
& A1-3 & 20.9 & 20.9 & 2.3 & 1.57 & 0.199 \\
& A1-4 & 20.7 & 20.8 & 2.2 & 1.55 & 0.209 \\
& A1-5 & 20.8 & 20.7 & 2.2 & 1.54 & 0.210 \\
A2 & A2-1 & 21.2 & 20.9 & 5.5 & 3.90 & 0.205 \\
& A2-2 & 21.1 & 21.0 & 5.4 & 3.90 & 0.208 \\
& A2-3 & 21.0 & 21.1 & 5.4 & 3.93 & 0.209 \\
A3 & A3-1 & 21.3 & 20.9 & 10.6 & 7.81 & 0.209 \\
& A3-2 & 21.0 & 21.3 & 10.6 & 7.83 & 0.210 \\
& A3-3 & 21.1 & 21.0 & 10.8 & 7.84 & 0.209 \\
A4 & A4-1 & 21.3 & 21.1 & 21.6 & 15.71 & 0.206 \\
& A4-2 & 21.0 & 21.2 & 21.6 & 15.68 & 0.208 \\
& A4-3 & 21.1 & 21.0 & 21.7 & 15.68 & 0.208 \\
A5 & A5-1 & 21.1 & 21.0 & 43.2 & 31.40 & 0.209 \\
& A5-2 & 21.1 & 21.0 & 42.5 & 31.40 & 0.212 \\
& A5-3 & 21.1 & 21.0 & 43.2 & 31.39 & 0.209 \\
\hline
\end{tabular}

Table 3 The parameters of cuboid samples with different base cross sections

\begin{tabular}{llllllll}
\hline Set & Specimen name & Width $/ \mathrm{mm}$ & Depth $/ \mathrm{mm}$ & Thickness $/ \mathrm{mm}$ & Mass/g & Relative density & Cross-sectional area/mm ${ }^{2}$ \\
\hline B1 & B1-1 & 10.9 & 20.5 & 20.0 & 7.47 & 0.212 & 223.45 \\
& B1-2 & 10.9 & 20.5 & 20.0 & 7.52 & 0.213 & 223.45 \\
B2 & B2-1 & 20.9 & 21.0 & 20.0 & 14.62 & 0.212 & 438.90 \\
& B2-2 & 21.0 & 21.0 & 20.0 & 14.61 & 0.213 & 441.00 \\
\hline
\end{tabular}




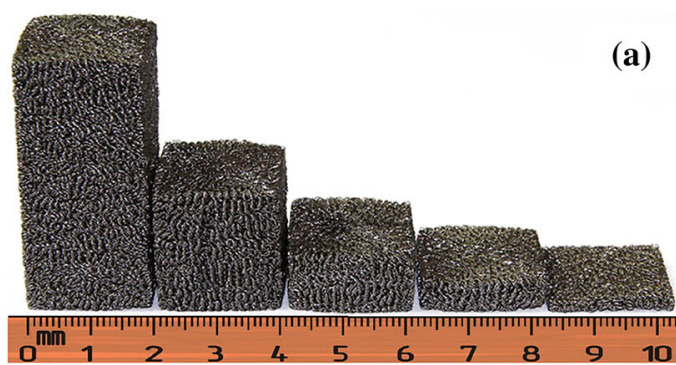

(d)

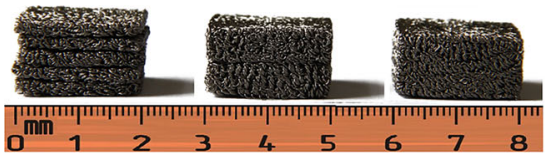

Figure 1 The EMWM samples used for the tests. Specimens with a different thicknesses and, $\mathbf{b}$ different base cross sections, c samples connected in parallel, d combination of EMWM specimens in series and a single EMWM with similar total

loading. The tangent modulus is represented by the slope of the stress-strain curve at any specific strain point. Below the proportional limit, the tangent modulus is equivalent to the Young's modulus and can be expressed by the following equation [16]:

$E_{T}=\frac{\Delta \sigma}{\Delta \varepsilon}=\frac{\Delta F / A}{\Delta z / H}$

In (2), $A$ is the cross-sectional area of the sample, $H$ is the initial height (thickness) of the EMWM sample, the compression force is $\Delta F$, and the displacement under compressive loading is $\Delta z$. The energy dissipated during one loading-unloading cycle is the difference between the areas under the loading and unloading curves, and can be indicated as $\Delta W$ (Fig. 2a). The maximum energy stored during a cycle, $U$, can be calculated from the area under the middle line of the hysteresis loop (Fig. 2b). The energy dissipation coefficient (or loss factor) is therefore given by the following relation [16, 26, 27]:

$\eta=\frac{\Delta W}{\pi U}$

The compressive tests have been carried out using a WDW 3100 electronic universal testing machine (Fig. 3a), with a $2.5 \mathrm{kN}$ force sensor (accuracy of $4 \%$ ) and a $25 \mathrm{~mm}$ electronic dial gage to measure the load and deformation (Fig. 3b). Each specimen was subjected to an initial pre-stress of $12.5 \mathrm{kPa}$ to decrease the influence of the uneven contact occurring at small deformations between the EMWM specimen and the (b)

(c)
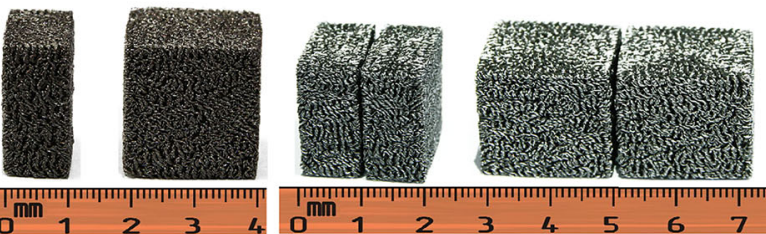

(e)

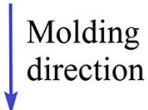

thickness, e photo and schematic diagram related to five EMWM samples connected in series and separated by steel plates. The blue arrow indicates the molding direction during the manufacturing and the loading direction during testing.
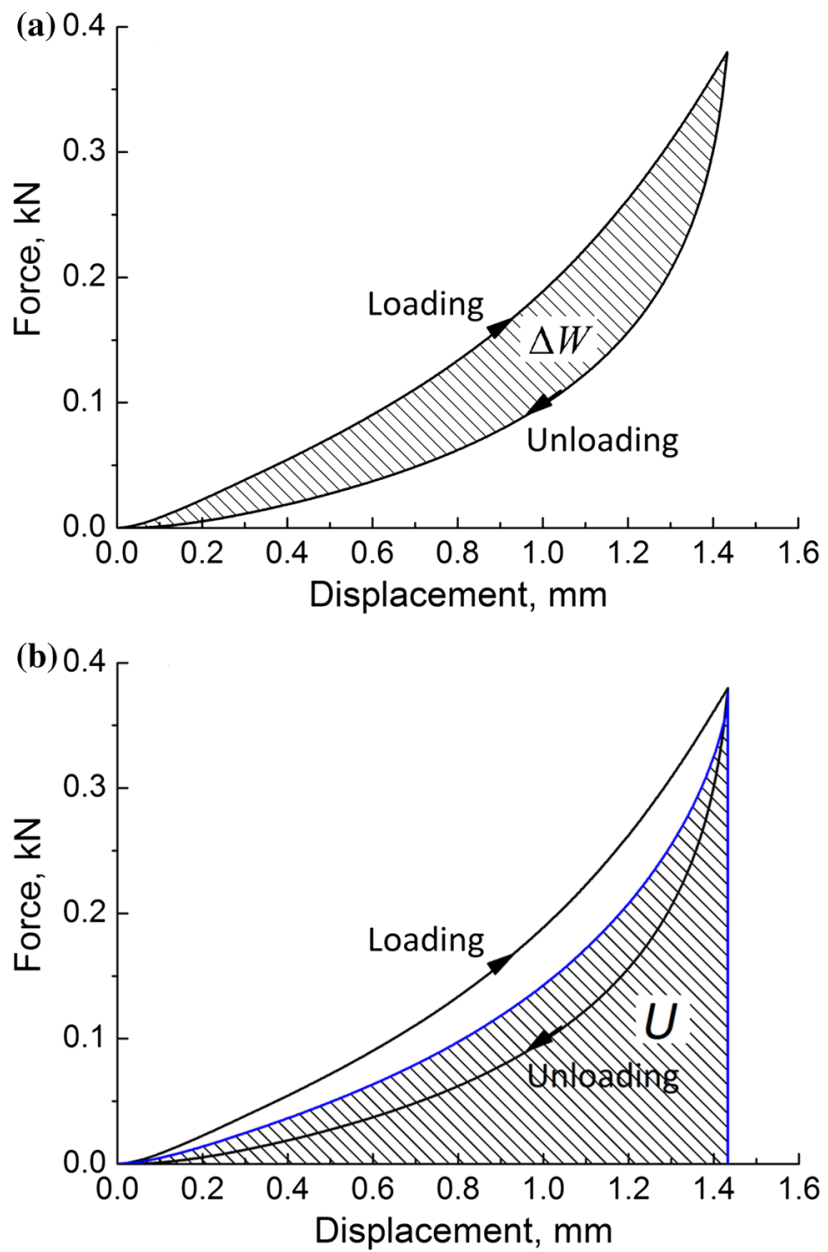

Figure 2 Graphical representation of a energy dissipated $\Delta W$ and b energy stored $U$ during a single cyclic loading, used in Eq. (3) [16]. 

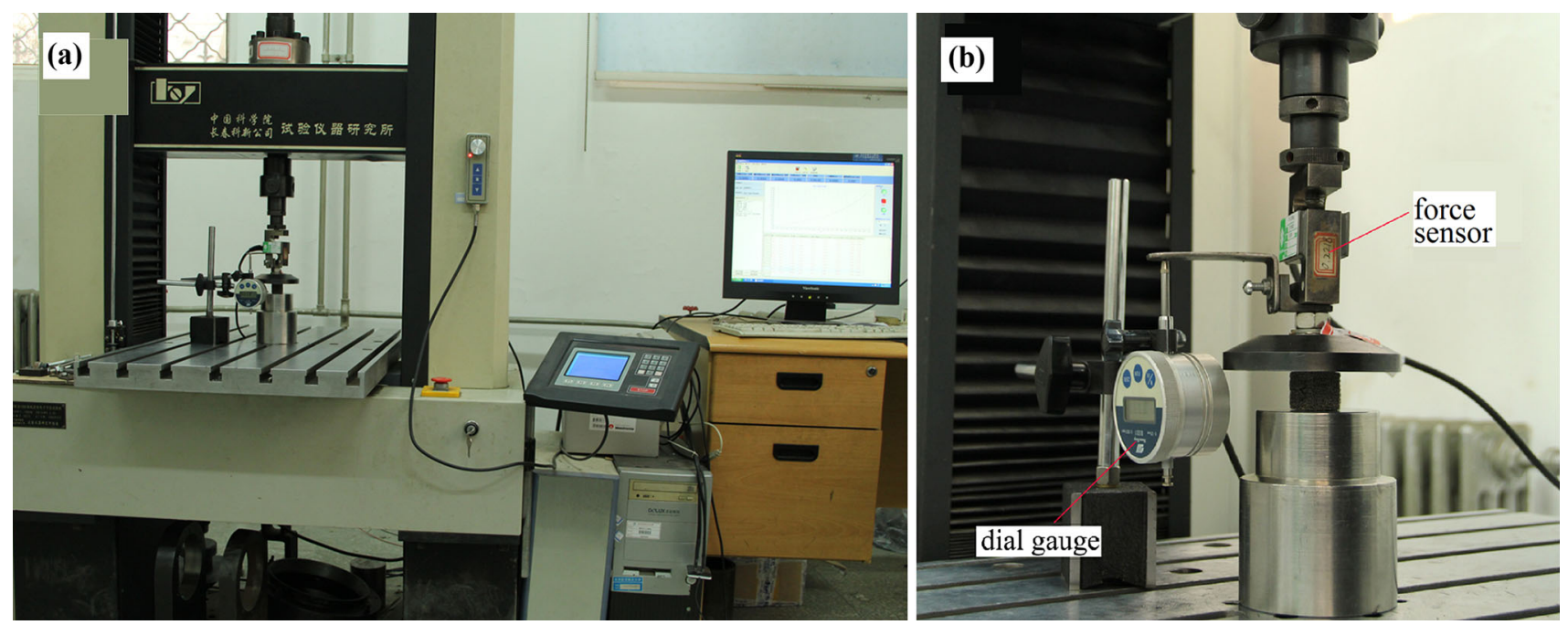

Figure 3 Test rig, a electronic universal testing machine and $\mathbf{b}$ force sensors and electronic dial gage used in the experiments.

holders. All the specimens were tested using a strain rate of $0.0033 \mathrm{~s}^{-1}$.

Figure 4 shows a typical example of the compressive force-displacement curves. After two compressive cycles, the mechanical behavior of the EMWM sample appears to be stabilized. The difference between the first two cycles is mainly caused by the uneven contact surface between the EMWM samples and the holders. Neither the top surface nor the bottoms of the EMWM specimens are absolutely flat, and this results in the presence of an unstable and irregular contact reaction force between the EMWM materials and holders. After two cycles, the distribution of the entangled wires and the contact status at the surface appear however to be satisfactory. This

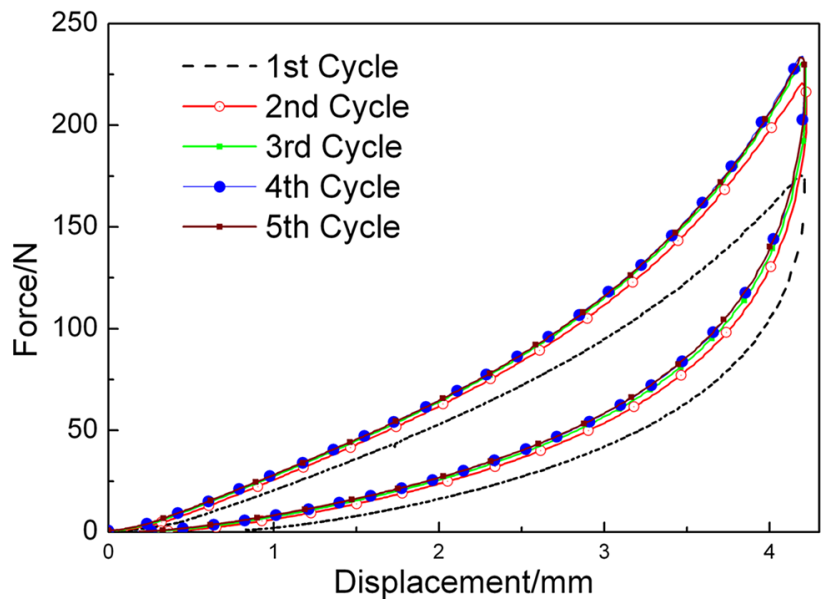

Figure 4 Representative cyclic force-displacement curves for the A4-1 sample. phenomenon has also been reported in [16] for EMWM solids and in [28] for open cell PU foams. In real engineering applications, EMWM solids will generally undergo thousands of loading cycles after installation, and the mechanical behavior during the initial unstable cycles does not affect significantly the operational performance of the system. Therefore, in this work, only the mechanical properties acquired from the 5th cycle have been taken into consideration as being representative of the EMWM solids. The loading part of the 5th cycle curve is used to obtain the tangent modulus [16]. The experimental data are initially converted into a stress-strain curve according to Eq. (2) (see the black dots in Fig. 5a), and a sixth-order polynomial fitting is then used to fit these points (red curve in Fig. 5a). The tangent modulus (Fig. 5b) is obtained from the gradient of the polynomial-fitted curve. A similar approach is also adopted to identify the loss factor, i.e., the stressstrain curves for both the loading and unloading parts are fitted first, and then the middle Riemann sum method is employed to calculate the related area of $\Delta W$ and $U$.

\section{Results}

\section{Compression mechanics of the EMWMs}

The test results indicate that all EMWM specimens always exhibit a similar mechanical behavior. Figure 6 shows, as an example, the results obtained by the three samples belonging to the A4 set. The 

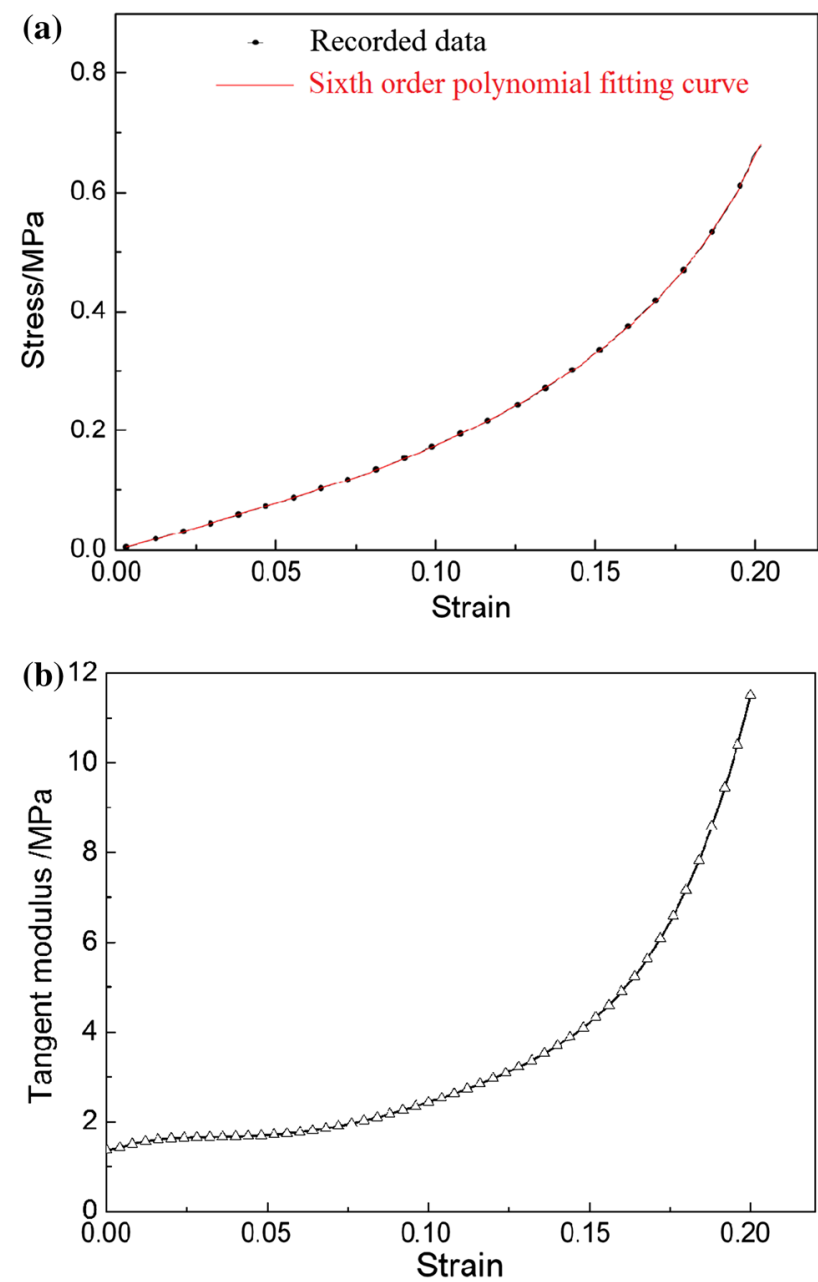

Figure 5 Data processing and methodology to obtain the tangent modulus for the case sample A4-1, a recorded data and polynomial fitting result for the stress-strain curve, and $\mathbf{b}$ tangent modulusstrain curve.

EMWM material appears to exhibit a three-stage tangent modulus-strain behavior. At small strains (up to $2.5 \%$ ), it is possible to observe an initial increase (Fig. 7a), followed by a quasi-constant region for increasing strains (up to $5 \%$, Fig. $7 b$ ). From this point onwards, the tangent modulus increases significantly and exhibits a strong straindependent behavior (Fig. 7c). Within the quasi-constant region, the value of the standard deviation (average $3.3 \%$ of the mean) is the smallest, and the EMWM samples exhibit the most stable properties.

The presence of three stages during the compressive loading could be explained by using the microstructural configurations defined in the EMWM
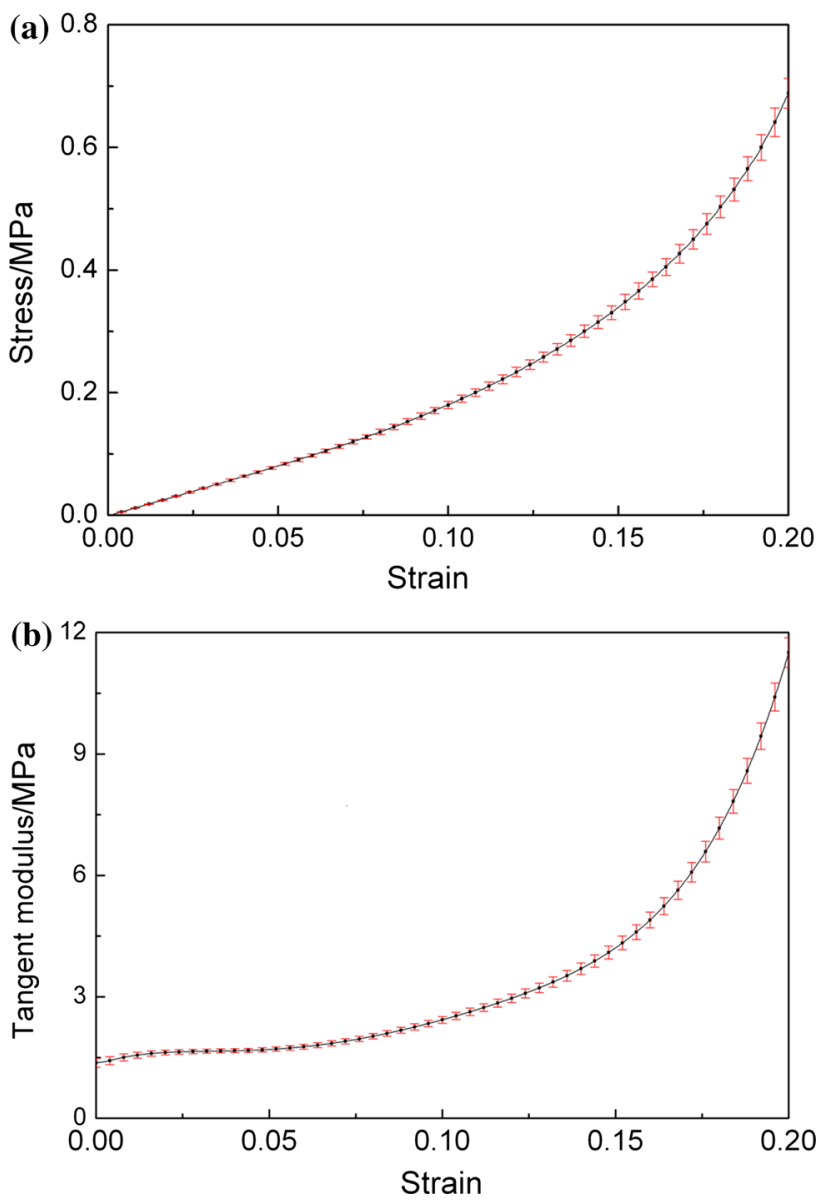

Figure 6 The results for the A4 batch. a Stress-strain curve with standard deviation obtained by the polynomial fitting, and b tangent modulus-strain curve with standard deviation calculated from the above stress-strain curve.

spiral wire model $[9,29]$. At small strains, the EMWM specimen is subjected to a non-uniform distribution of forces due to the uneven top surface of the sample. Only parts of the wires are therefore compressed and deformed, although the compressive stress will increase for increasing deformation, with consequent increase of the tangent modulus. During the following loading stage, almost all the helix wires are compressed. The elastic deformation of the wires coupled with a minimal component of relative slip leads to the quasi-constant tangent modulus observed experimentally. For increasing deformations, the level of entanglement between wires increases significantly, resulting in an equivalent densification and more "stick" interaction, and therefore the tangent modulus increases rapidly within the final strain region. 

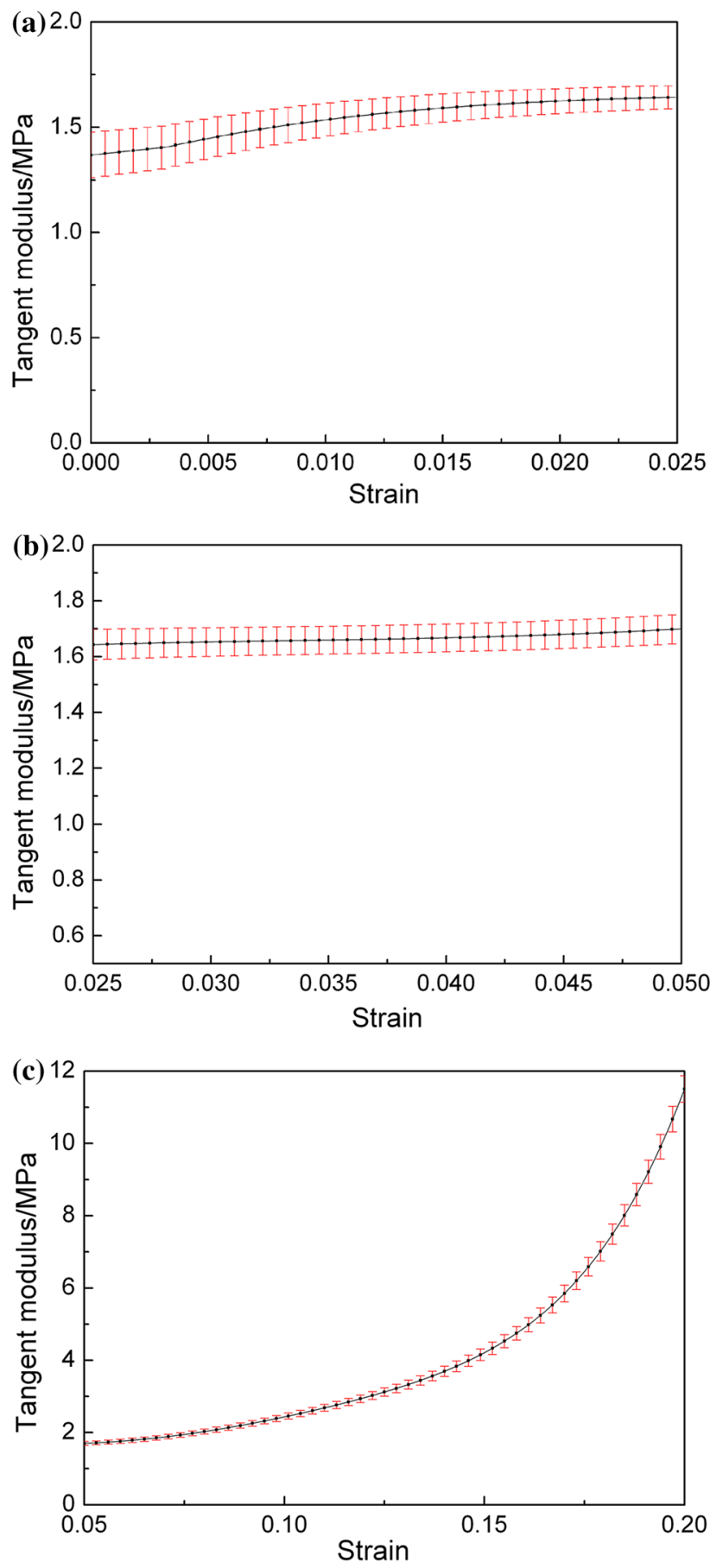

Figure 7 The three stages of the tangent modulus-strain curves for a small strains at less than $0.025, \mathbf{b}$ within the quasi-constant region at from 0.025 to 0.050 , and $\mathrm{c}$ final rapid densification at greater than 0.050 .

\section{The influence of cross section and parallel connection}

Samples with different rectangular cross sections (but substantially equal thickness) have been

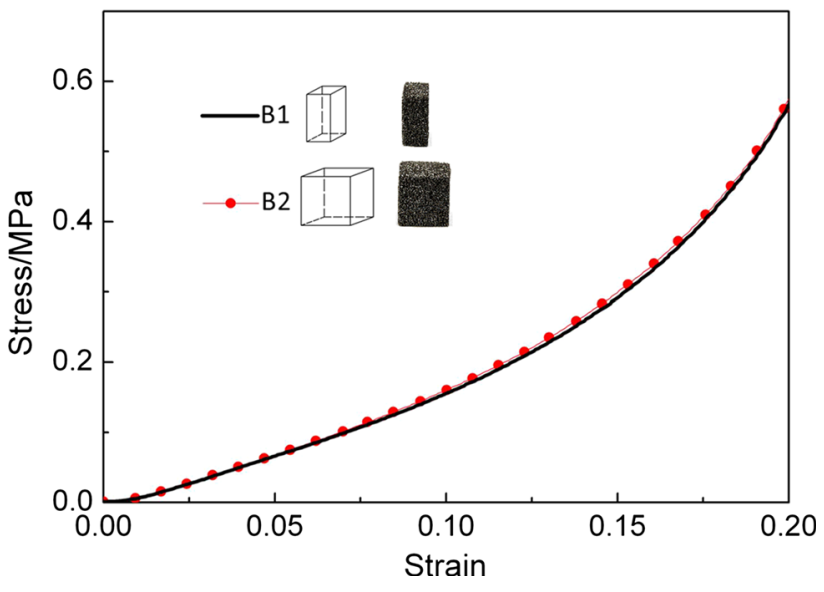

Figure 8 Comparison between stress-strain curves of samples with different cross-sectional areas $(11.0 \mathrm{~mm} * 20.5 \mathrm{~mm}$ for the B1 batch and $21.0 \mathrm{~mm} * 21.0 \mathrm{~mm}$ for the B2 set).

manufactured and tested (Fig. 1b), and their parameters are listed in Table 3. Figure 8 shows the stressstrain compressive behavior of the specimens with the different sectional areas. The samples exhibit similar stress-strain curves, and the different surfaces do not show any clear influence on the mechanical properties of the EMWMs. The cuboid specimens were also tested under cyclic loading in a parallel connection topology, to identify the possible effects on the hysteresis of the material tested (Fig. 1c). In a parallel connection layout, the two EMWM samples showed very similar mechanical properties (Fig. 9a, b).

\section{The influence of the thickness for single EMWM specimens}

The compressive tests have been performed on cuboid samples with different thicknesses $H$ (see Table 2). For each thickness, we have evaluated at least three samples. The average stress-strain and tangent modulus-strain curves are shown in Fig. 10. The results show the significant influence exerted by the thickness over the compressive properties of the EMWM samples. For a thickness range between 2.2 and $10.6 \mathrm{~mm}$, the tangent modulus obviously increases for increasing values of the thickness (Fig. 10b). For samples thicker than $10.6 \mathrm{~mm}$, the mechanical performance is, however, almost unchanged. Table 4 shows some specific values of the average tangent modulus at different strains. The loss factors calculated for maximum strains of 0.20 for different thicknesses samples are shown in Fig. 11. One can observe an evident reduction of the loss 

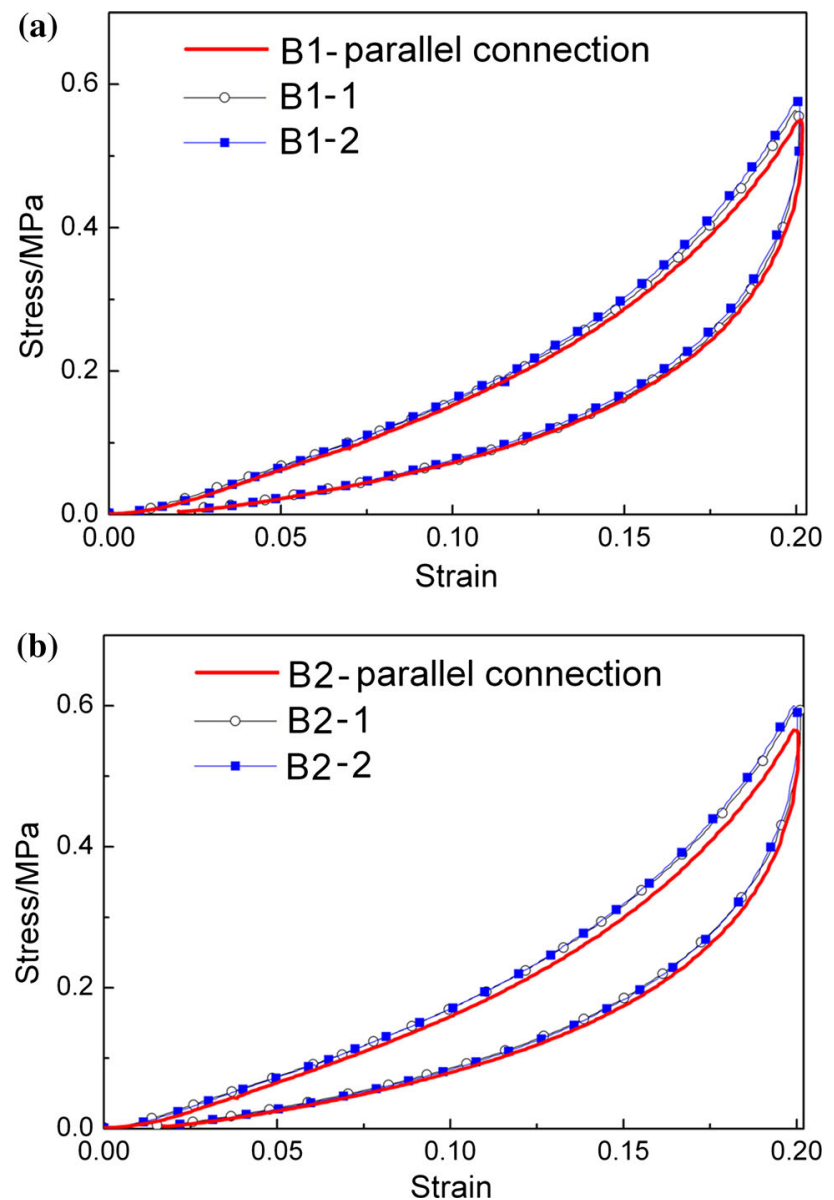

Figure 9 Cyclic stress-strain curves for the EMWMs in single and parallel connection. Results for the $\mathbf{a} \mathrm{B} 1$ and $\mathbf{b}$ B2 sets.

factors for increasing thickness values, with a $27 \%$ drop when passing from a thickness of $2.2 \mathrm{~mm}$ to the largest one of $42.8 \mathrm{~mm}$.

\section{Connectivity in series}

\section{EMWM-EMWM structures with similar total thicknesses}

Three different EMWM sample structures with similar total thicknesses (about $10.8 \mathrm{~mm}$ ) but made from different numbers of individual EMWM laminae as shown in Fig. 1d have been tested. The results relate to the tangent modulus are shown in Fig. 12. The combination of the EMWM laminae in series gives a relative low value of the tangent modulus at strains smaller than 0.08. Above that level of deformation, the tangent modulus of the different EMWM systems is all quite similar. The behaviors of the strain-dependent loss factor for the different EMWM combinations are shown in Fig. 13. Contrary to the case of the tangent modulus, the loss factor of the different
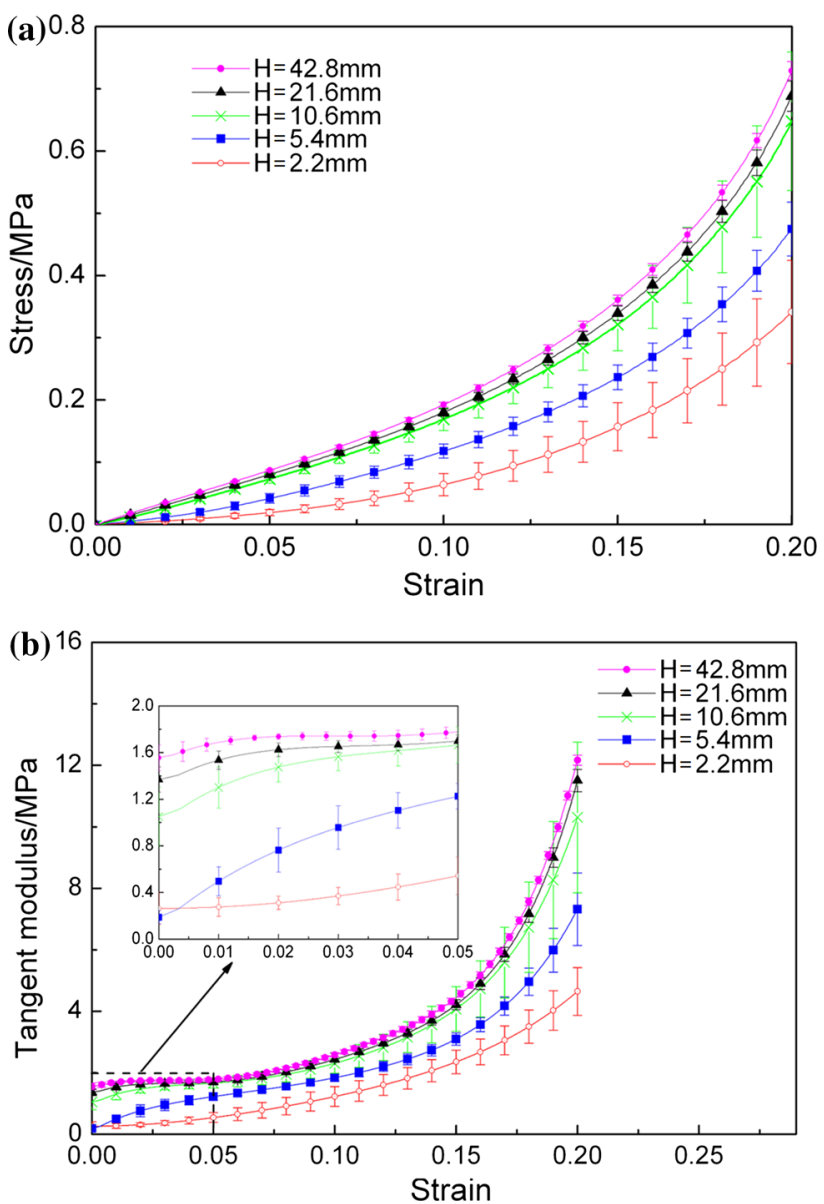

Figure 10 Results from the samples with different initial thicknesses. a Average stress-strain curves with standard deviation; b tangent modulus-strain curves with standard deviation. The region representing the strain from 0.00 to 0.05 is locally zoomed in.

EMWM series layers changes significantly with the sample architecture. It is worth noticing that the combination with two 5.4-mm-thick laminae shows slightly higher loss factors compared to the single 2.2mm-thick EMWM plate, with loss factors ranging between 0.14 and 0.20 for compressive strains up to $10 \%$. Within the same strain range, the EMWM combination made from five $2.2 \mathrm{~mm}$ laminae has significantly higher loss factors (between 17 and $28 \%$ ), closer to the ones shown by the whole 10.7mm-thick sample. For strains higher than $10 \%$, the different systems tend however to converge to similar values $(0.22$ at $20 \%$ of strain).

\section{EMWM-steel structures}

Another EMWM system designed for specific EMWM engineering applications is represented by 
Table 4 Tangent modulus for samples with different thicknesses

\begin{tabular}{|c|c|c|c|c|c|}
\hline \multirow[t]{2}{*}{ Set } & \multirow[t]{2}{*}{ Average thickness $/ \mathrm{mm}$} & \multicolumn{4}{|c|}{ Average tangent modulus/MPa } \\
\hline & & Strain at 0.05 & Strain at 0.10 & Strain at 0.15 & Strain at 0.20 \\
\hline A1 & 2.2 & 0.543 & 1.227 & 2.351 & 4.644 \\
\hline A2 & 5.4 & 1.225 & 1.839 & 3.098 & 7.319 \\
\hline A3 & 10.6 & 1.664 & 2.309 & 4.070 & 10.306 \\
\hline A4 & 21.6 & 1.699 & 2.432 & 4.209 & 11.505 \\
\hline A5 & 42.8 & 1.779 & 2.583 & 4.442 & 12.167 \\
\hline
\end{tabular}

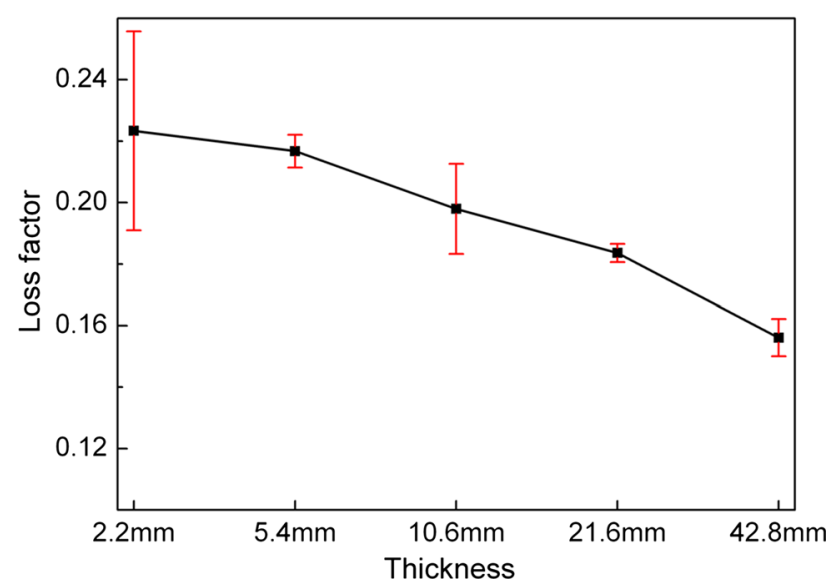

Figure 11 Loss factors for different thicknesses at a compressive strain of 0.2 .

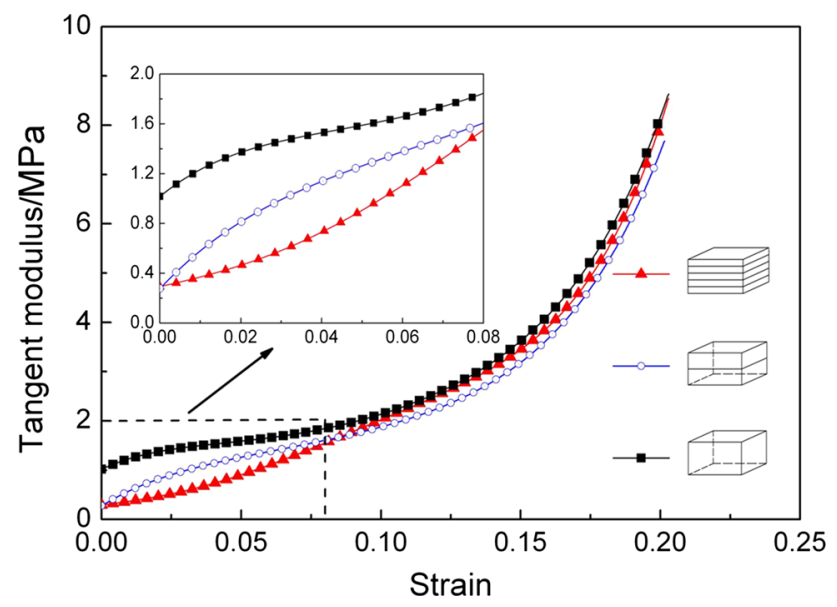

Figure 12 Comparison between different structures with similar total thicknesses $(\sim 10.8 \mathrm{~mm})$. The EMWM systems are made from a combination of 5 EMWM specimens and 2 EMWM specimens in series, and a single EMWM. The region for strain from 0.00 to 0.08 is locally zoomed in.

entangled metal wire laminae connected in series with interleaving steel plates (Fig. 1e). The steel lamina represents a hard surface similar to the contact between the EMWM samples and tensile

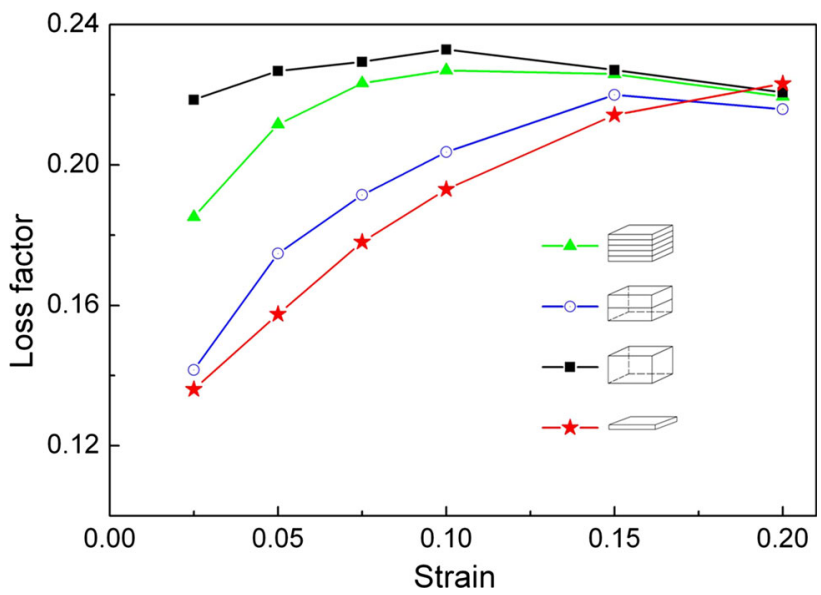

Figure 13 Loss factors for 5 EMWM specimens in series with a total thickness equal to $10.8 \mathrm{~mm}, 2$ EMWM specimens in series with total thickness equal to $10.8 \mathrm{~mm}$, and two single EMWMs with different thicknesses (10.8 and $2.2 \mathrm{~mm}$ ).

machine holders. The thin steel plate at the same time impedes the direct contact between neighboring EMWM laminae. The stress-strain curve for this case is represented in red in Fig. 14, compared with the single EMWM (black) and the samples connected in series without steel laminae (blue).

When the steel laminae are applied to isolate the direct contact between the EMWMs the peak, stress decreases, and the stress-strain curve is almost the same as the one of a single EMWM lamina. This is an indication that the increase of the peak stress in the EMWM laminae connected in series occurs when there is interface contact between EMWM solids, but not between the EMWM and the grip holders.

\section{EMWM-EMWM structures with similar EMWM elements}

Different numbers of EMWM samples from the A1 set (thickness is $2.2 \mathrm{~mm}$ for each) have also been combined in series and tested. The system in the left of Fig. 1d 


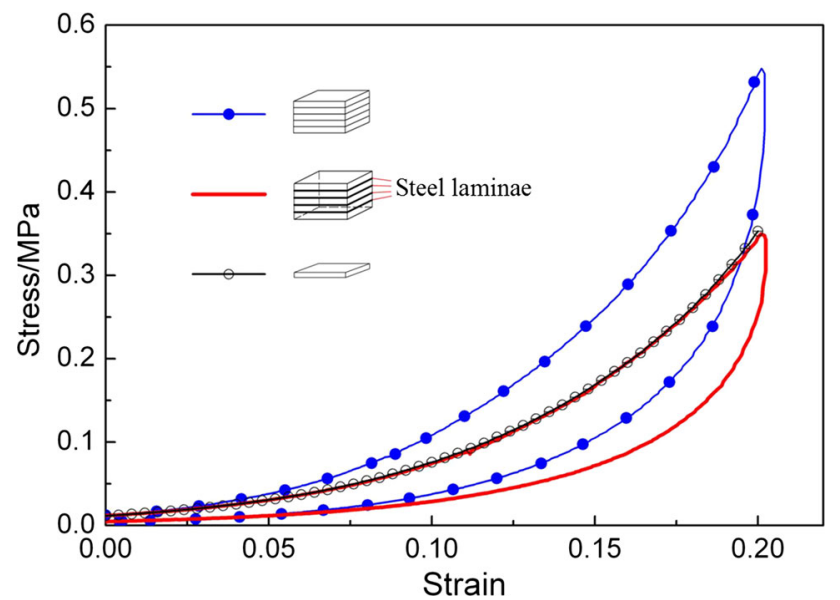

Figure 14 Stress-strain curves for samples connected in series without the steel lamina (total thickness $=10.8 \mathrm{~mm}$ ), samples connected in series insulated by the steel laminae (total thickness $=12.8 \mathrm{~mm}$ ), and single EMWM with thickness equal to $2.2 \mathrm{~mm}$

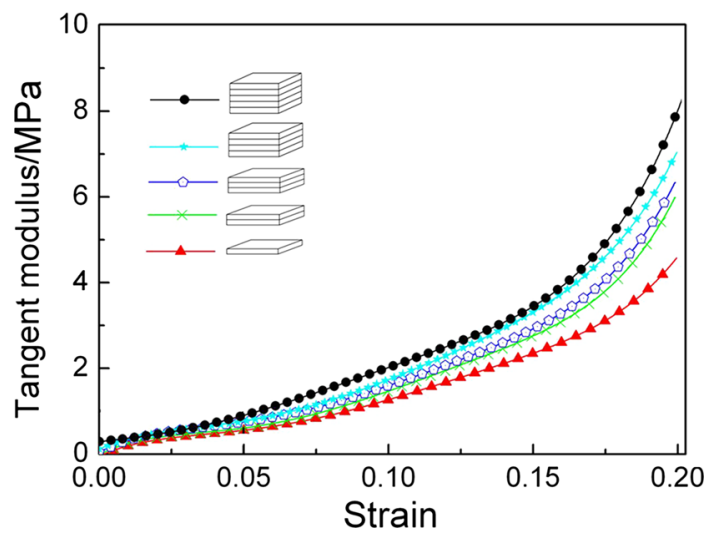

Figure 15 Tangent modulus-strain curves for combinations of different numbers of EMWMs in series. The parameters of each system are described in Table 5.

represents five EMWM laminae connected in series, and the tangent modulus-strain curves of the cases related to 1 and up to 5 layers are shown in Fig. 15.

The tangent modulus increases for increasing numbers of layers connected in series. This result is similar to the one related to single EMWM specimen (Fig. 10b). Table 5 provides some specific values of the tangent modulus at specific strains.

\section{Discussions}

The results in "The influence of the thickness for single EMWM specimens" section show the significant influence provided by the sample thickness over the global mechanical compressive properties of entangled metal wire systems. "Connectivity in series" section also shows the peculiar behaviors observed in EMWM samples when connected in series. The various physical aspects underpinning these results may be explained by using the concept boundary layer (BL). The microscopic characteristics and deformation mechanisms of the boundary layers are first presented in this section, and the possible BL effects over the global properties of EMWM systems are then discussed.

\section{The boundary layer of a EMWM}

\section{Identification of boundary layer in the EMWM's microstructure}

When an EMWM specimen is fabricated, the weaving and molding processes always cause non-homogeneous distributions of the metal wire and of the deformation along the molding direction between the boundary and the inner part of the solid. The particular microstructure near the edge of an EMWM solid can be observed by a computed tomography scanning and slices are obtained by the software package "VGStudio MAX 2.1" (Fig. 16). The scan is obtained from one unloaded cylindrical EMWM specimen (height of $30.0 \mathrm{~mm}$ and cross-section diameter of $30.0 \mathrm{~mm}$ ). The slices are imaged at every $0.2-\mathrm{mm}$ interval to show the different cross sections at different axial positions of the sample. The surface is defined at the plane $X=0 \mathrm{~mm}$ ( $X$ represents the molding direction, i.e., also the axial direction of the cylinder sample). In Fig. 16, the shapes and the wire sections are displayed with solid lines, and they provide a qualitative indication of the different distributions for wires of the cross sections between the $\mathrm{BL}$ and the internal part of the sample. The presence of a densification of the wires toward the middle of the BL section is also apparent, while for the inner part the wire distribution is much more uniform.

The distributions of the wire cross sections of this EMWM sample across the molding axis are further evaluated in a quantitative manner by using the open source software "ImageJ 4.6." The total areas surrounded by the closed wire edges and the numbers of closed lines describing the wire sections are calculated by the code according to the following process. Firstly, an appropriate set scale is given for the image; the value is 87.7 pixels $/ \mathrm{mm}$ for this particular case, 
Table 5 Tangent modulus for the EMWMs in series with different numbers of layers

\begin{tabular}{|c|c|c|c|c|c|}
\hline \multirow[t]{2}{*}{ The number of layers in series } & \multirow[t]{2}{*}{ Thickness/mm } & \multicolumn{4}{|c|}{ Tangent modulus/MPa } \\
\hline & & Strain at 0.05 & Strain at 0.10 & Strain at 0.15 & Strain at 0.20 \\
\hline 1 & 2.2 & 0.594 & 1.230 & 2.382 & 4.655 \\
\hline 2 & 4.4 & 0.611 & 1.473 & 2.759 & 5.987 \\
\hline 3 & 6.6 & 0.754 & 1.600 & 2.927 & 6.338 \\
\hline 4 & 8.8 & 0.769 & 1.738 & 3.321 & 7.033 \\
\hline 5 & 10.8 & 0.905 & 2.021 & 3.441 & 8.040 \\
\hline
\end{tabular}
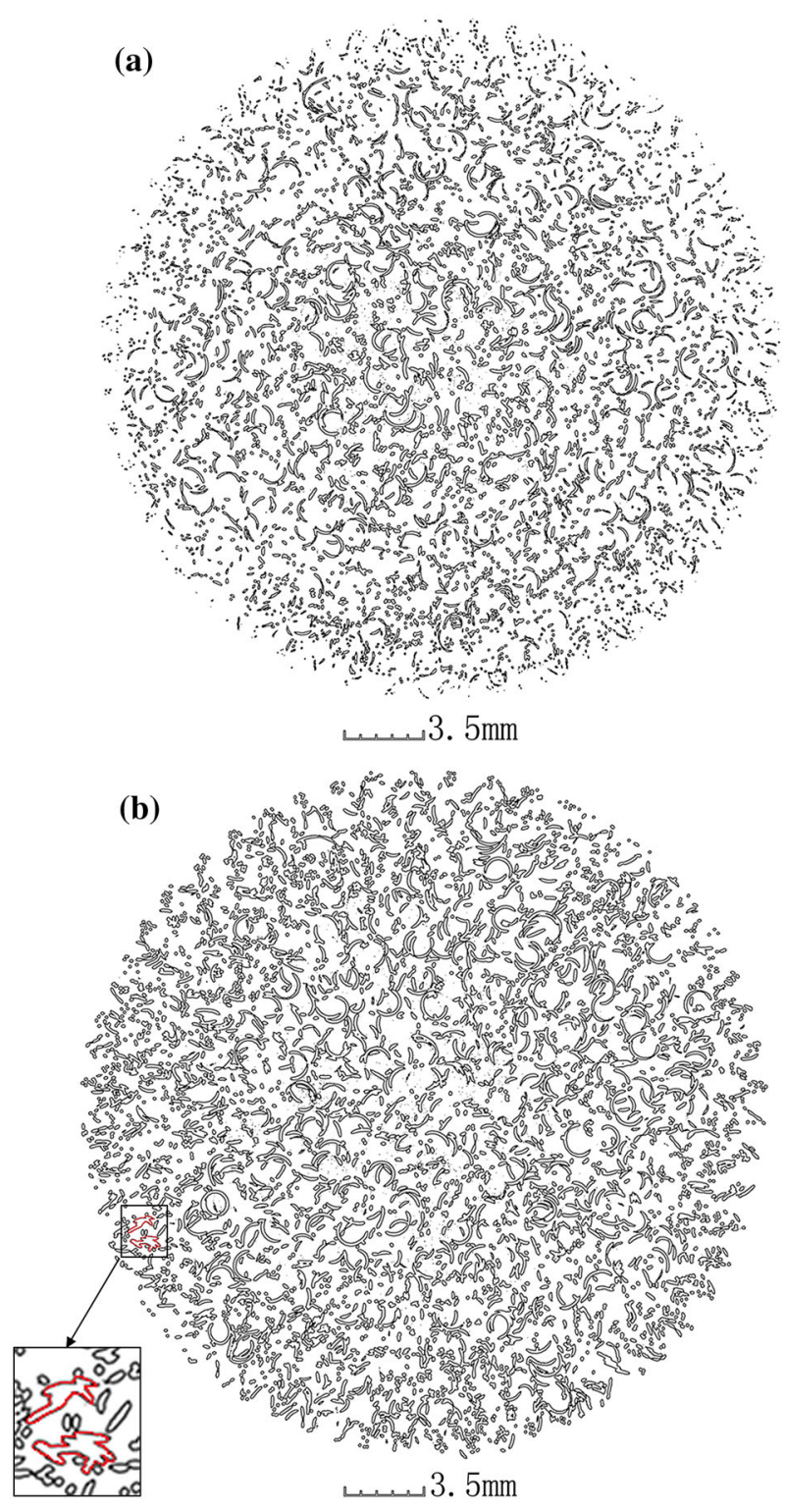

which is determined by the resolution ratio of the image. The picture is then converted to an 8-bit type (i.e., black and white format), and the "Huang" method is used to determine the threshold value for adjusting the brightness. Finally, the size is defined as "from 0 to infinity $\mathrm{mm}^{2}$," and the circularity is as "from 0 to 1.00 " to analyze all the particles in the image. Figure 17 is obtained by repeating the above operations for each cross-section image.
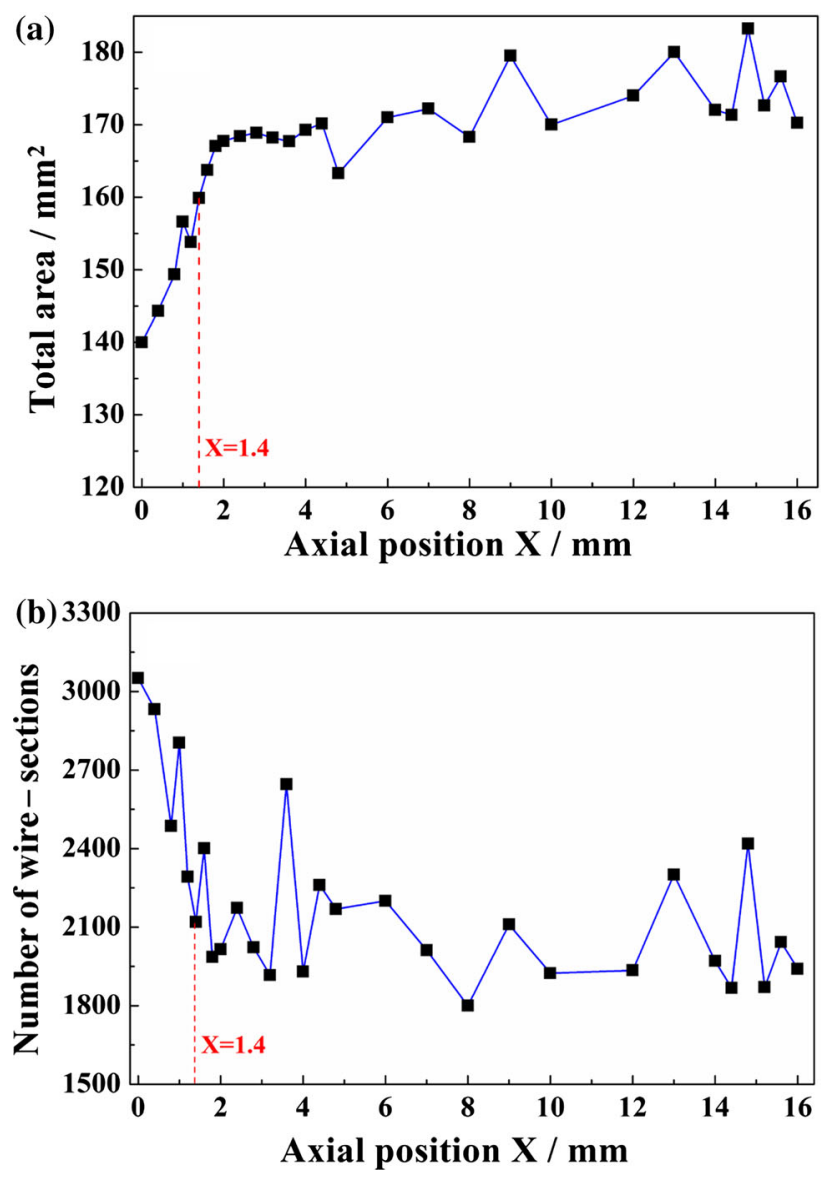

Figure 16 Cross section of a cylindrical EMWM sample obtained by X-ray tomography, the thickness of each slice being $0.2 \mathrm{~mm}$. Images for $\mathbf{a}$ the boundary layer at $\mathrm{X}=0.6 \mathrm{~mm}$, and $\mathbf{b}$ the internal section at $\mathrm{X}=15.0 \mathrm{~mm}$ with a local part zoomed in.

Figure 17 The distribution of wire cross sections at different axial positions. a Total area occupied by the wire sections per layer; b number of wire sections per axial location. 

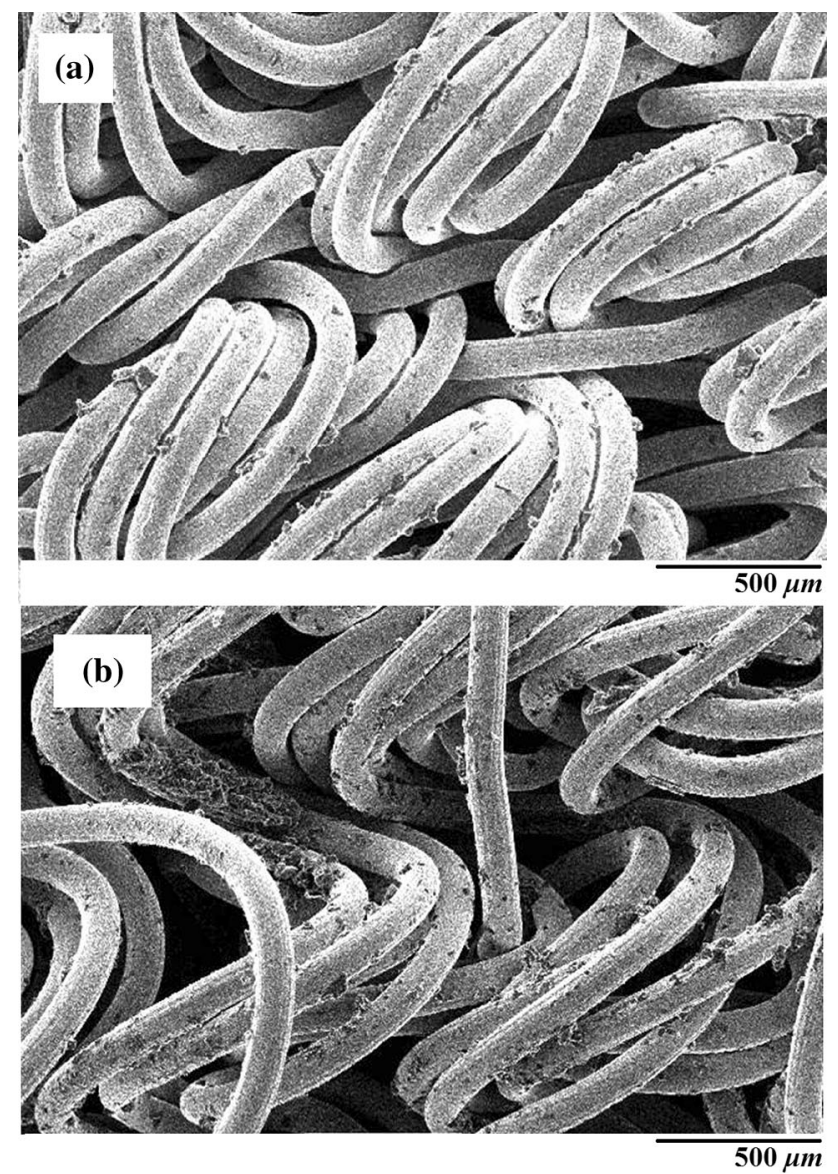

Figure 18 SEM images of the microstructure for a the internal part and $\mathbf{b}$ the boundary layer.

Figure 17 shows the distribution of the wire sections within the $\mathrm{BL}$ (i.e., the region between $X=0.0 \mathrm{~mm}$ and approximately $\mathrm{X}=1.4 \mathrm{~mm}$ ). The distribution is different from the one in the central portion of the sample. From Fig. 17a, one can observe a relatively small total area occupied by the wire sections in the BL, which indicates the lower presence of wire solids in the BL than in the inner parts. Figure $17 \mathrm{~b}$ also shows that there are a lower number of wire sections in the inner part. This fact can be explained by considering that it is difficult to distinguish the contact between adjacent wires by using the above low-resolution CT images and the related postprocessing method. Therefore, when two or more wires are in contact, the software will regard them as a one single wire section in most cases, due to the single set of closed lines. An example of this is represented in Fig. 16b, in which two sets of wire delimited by the red closed lines are shown in the local zoomed figure. Each set of closed lines is actually composed by more than one wire; however, the software regards them as one single wire section; the lower number of wire sections therefore possibly represents the more existence of the contacted wires. In other words, a larger number of wire sections in the boundary layer indicate that there are more wires detached in the BL part. From the above data, we postulate that there are less wire solids and contact pairs in the BL section, which indicates that the wires cannot sufficiently entangle in the boundary layer.

SEM images (Fig. 18) also corroborate the presence of a boundary layer of the MR sample. From the first observation of the two figures, one can notice more surface grooves (or gaps) running parallel with the wire axes in the BL part (Fig. 18b). The existence of the grooves is caused by the recovering deformation of the wires when the molding force is unloaded during the sample manufacture. As mentioned in "EMWM specimens" section, the molding is the last process to produce the sample, and then the specimen is extracted from the mold when the size of the sample along the molding direction will increase about $50 \%$ [30] due to the elastic rebound deformation of the wires. When the wires of the inner part tend to rebound, they will interact with other more wires compared to the ones in the BL. As a consequence, more gaps will be filled. The wires in the BL can, however, deform with more freedom, creating therefore more gaps. The wires in the BL part occupy as a consequence a lower total area, and when the sample is subjected to compression again less wires will behave under stick or slip. When we look along the direction of the wires in the SEM images (Fig. 18b), we can observe that most of the helixes appear to be aligned along the longitudinal direction, leading to a larger number of wire sections, but a smaller single wire-section area. A lower total area is therefore taken up by the wires present in the BL parts. It is also worth to notice that the results from both the CT scans and the SEM show similar features at both ends of the specimens, although the topology of only one end is discussed in this paper.

\section{The deformation behavior of the boundary layers}

When the EMWM samples are compressed, the wires located in the internal part of the sample tend to experience a high level of entanglement with mainly slip and stick contact interactions. Within the BL region, the wires possess however a small number of 
adjacent wires (as in Figs. 16, 17). The BL wires when compressed tend to enter into contact with the tensile machine holders, or further penetrate the rest of the EMWM solid. Figure 19 provides a sketch illustration of the Boundary Layer topology under compression. The two boundary layers have similar features, i.e., the contact occurs between the EMWM sample and the holder (or the steel plate), and we denominate this type of contact as soft-to-hard BL (SHBL). There is, however, another type of boundary layer, with contact interfaces existing between the EMWM samples (i.e., soft-to-soft BL-SSBL).

The SHBLs will exhibit smaller tangent modulus than the inner region of the sample for the whole loading strain range. This fact can be explained by considering the EMWM spiral wire model [9, 29, 31]. The wires in the EMWM solid can have different types of interaction during compression (non-contact, slip, and stick-Fig. 20), and the effective stiffness for slip or stick is much higher than that for non-contact. As indicated in Figs. 16 and 17, there is smaller total

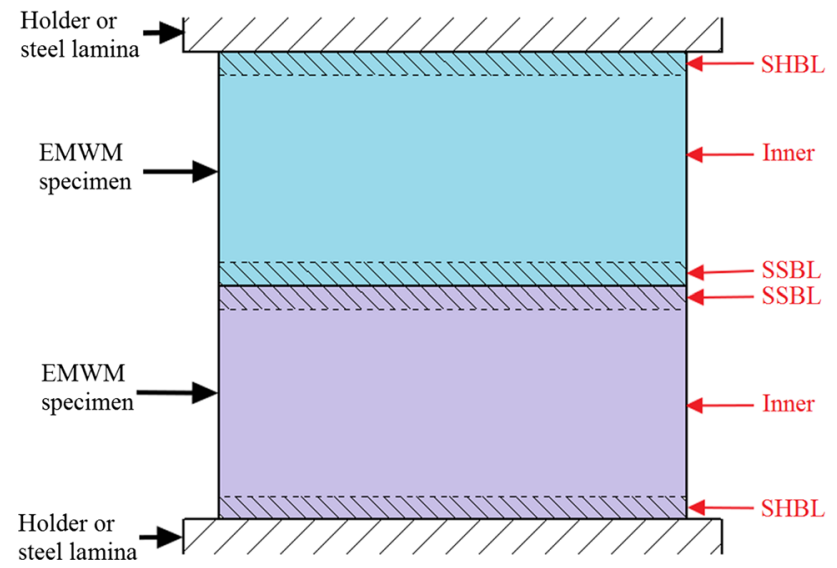

Figure 19 Sketch illustration of the different types of boundary layers. area and larger number for the cross sections of the wires, which means the non-contact status occupies larger percentage for the BL part than the inner part, leading to larger compliance, and a consequent decrease of the tangent modulus.

For the BLs with soft-to-soft contact ("SSBL"), the BL-related region will exhibit a smaller tangent modulus than the one present in the inner part of the sample at small strains, but the tangent modulus in the BL region will be close to the one of the inner part at large strains. The reason for this behavior is that with the increasing of the compression load, the wires related to the BL parts will tend to penetrate the adjacent EMWM solid, and higher numbers of wires previously not in contact will undergo a slip or stick behavior compared with the ones in the soft-to-hard BLs. At large strains, the tangent modulus of the softto-soft BLs will therefore reach similar value as the ones of the inner part of the porous material.

From the above considerations, we could produce a qualitative but physically consistent model of the effective tangent modulus inside the BL parts. If the modulus for the uniform inner parts of the EMWM specimens is assumed as $E_{1}$ at small strains and $E_{2}$ at large strains, the modulus for the BL parts could be qualitatively expressed as shown in Table 6.

Table 6 Qualitative relations for the tangent modulus in the EMWM boundary layers

\begin{tabular}{lll}
\hline & \multicolumn{2}{l}{ Tangent modulus } \\
\cline { 2 - 3 } & At small strains & At large strains \\
\hline Inner part & $E_{1}$ & $E_{2}$ \\
Soft-hard BL & $<E_{1}$ & $<E_{2}$ \\
Soft-soft BL & $<E_{1}$ & $\approx E_{2}$ \\
\hline
\end{tabular}

Figure 20 Three types of interaction during compression according to the EMWM micromechanics model: a noncontact, b slip, and c stick [31].

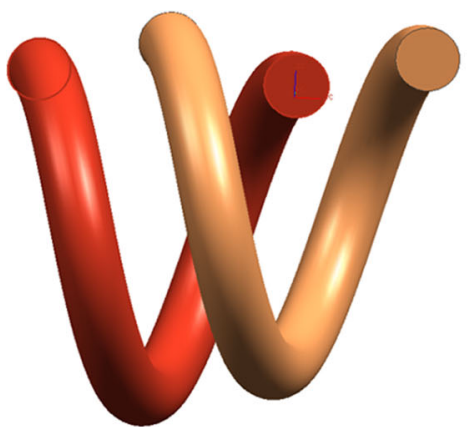

(a)

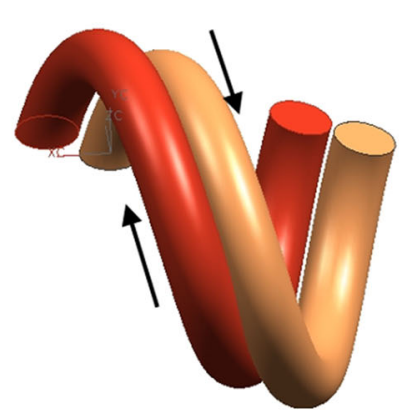

(b)

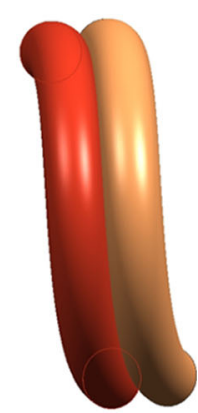

(c) 


\section{Effects of the boundary layer over the EMWM mechanical properties}

With the relations shown in Table 6, it is possible to provide explanations to the different experimental results shown in "The influence of the thickness for single EMWM specimens" and "Connectivity in series" sections.

\section{Size effect on single EMWM sample}

The size (thickness) effect described in "The influence of the thickness for single EMWM specimens" section produced by the BL can be understood by observing Fig. 21. The stiffness of the soft-hard BL is lower; therefore, the EMWM samples with a small total thickness will be affected more negatively by the boundary layer, leading to a lower global tangent modulus. For thicker samples, the influence of the BL is less pronounced, and samples thicker than $10.7 \mathrm{~mm}$ exhibit similar tangent modulus values.

\section{Stiffness relaxation effect on series connected EMWM samples}

If we now examine the results shown in Fig. 12 ("EMWM-EMWM structures with similar total thicknesses" section), the three EMWM systems have equal global sizes. As a consequence, the effects of the soft-hard BLs are the same but there are now different amounts of soft-to-soft boundary layers. Looking at the results in Table 6, one can notice that at small strains, the combination of the 5 EMWM laminae (i.e., the red curve in Fig. 12) shows the lowest tangent modulus because there are more softsoft BLs in this system, which lead to a more significant stiffness relaxation effect. For larger strains, the

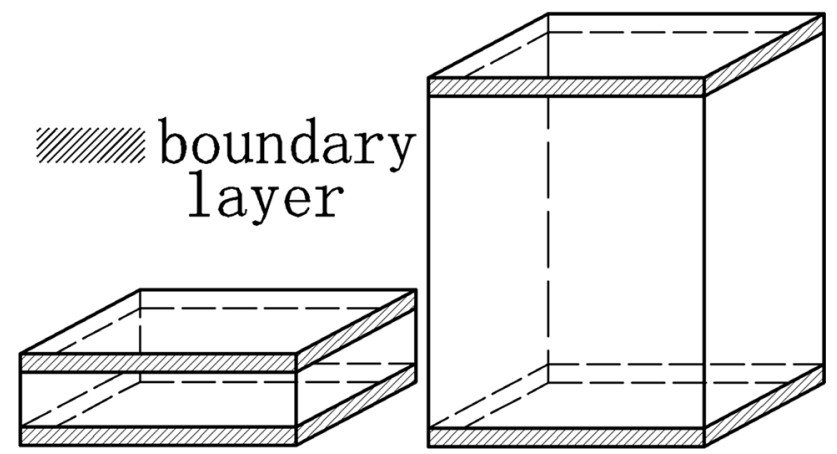

Figure 21 Scheme illustrating the scale size between boundary layers in the EMWM samples at different thicknesses. soft-to-soft BLs, however, exhibit similar stiffness comparing the inner parts (Table 6); therefore, the values of the tangent modulus are close for the three different structures.

The results shown in Fig. 14 show that the hysteresis loops for the single EMWM (black curve) and EMWMs with steel plates (red curve) exhibit the same behavior within the whole strain range. In this case, the steel plates represent the hard surfaces (similar to the tensile machine holders). The mechanical performance of the five EMWMs systems with steel laminae installed on the holders is completely equivalent to the one of one single EMWM. The tangent modulus for the five EMWMs without steel plates (blue curve) is however significantly higher than the one belonging to the system with steel laminae (red curve), in particular at large strains. Although the two systems possess equal global sizes and the same type of EMWM specimens, the former one (blue curve) involves the presence of 4 couples of soft-to-soft BLs rather than the soft-to-hard BLs of the latter one (red curve). As from Table 6, the former shows the higher stiffness of the two.

From the inspection of the results in Fig. 15 ("EMWM-EMWM structures with similar EMWM elements" section), the tangent modulus shows an increase when the total size is increasing. The phenomenon is quite similar to the one described in "The influence of the thickness for single EMWM specimens" section, and it appears that no evident effect is provided by the number of boundary layers. Table 6 shows that the stiffness relaxation effects provided by the soft-to-soft BLs could be neglected at large strains, within which only the dimension of the total size plays the most import role.

\section{Conclusions}

EMWM systems exhibit a three-stage tangent modulus-strain behavior under uniaxial compressive loading. Entangled metal wire material samples with different cross-sectional area show equivalent compressive properties, while the combination of two EMWMs connected in parallel shows similar stressstrain curves. The presence of the boundary layer in EMWM system is identified, and CT scanning gives evidence of the existence of BLs in which the microstructure of the wires and the local stiffness are different from the inner part. The boundary layer 
effect is responsible for the global size effect and stiffness relaxation effect on EMWM systems connected in series, resulting in the peculiar mechanical properties of the EMWM systems. The thickness of the samples has a significant influence on the global mechanical compressive properties. With an increase in thickness, the tangent modulus increases, but the loss factor diminishes for compressive strains up to $20 \%$. The EMWM systems connected in series exhibit lower tangent modulus and loss factors that depend on the architecture of the combined structure, the dimensions of the single EMWM solid, the global dimensions, and also different strain ranges.

These results are instrumental to design material systems based on EMWM with adaptive performance under different loading and geometric constraints.

\section{Acknowledgements}

This study was financially supported by the National Science Foundation of China (Grant Nos. 51475023 and 51475021), the UK Royal Society (Grant RG2611), and the Academic Excellence Foundation of BUAA for Ph.D. Students. The authors would also like to thank the anonymous reviewers and the editor for their constructive suggestions.

\section{Compliance with ethical standards}

Conflict of Interest The authors declare that they have no conflict of interest.

Open Access This article is distributed under the terms of the Creative Commons Attribution 4.0 International License (http://creativecommons.org/ licenses/by/4.0/), which permits unrestricted use, distribution, and reproduction in any medium, provided you give appropriate credit to the original author(s) and the source, provide a link to the Creative Commons license, and indicate if changes were made.

\section{References}

[1] Tan Q, He G (2013) Stretching behaviors of entangled materials with spiral wire structure. Mater Des 46:61-65

[2] Rodney D, Gadot B, Martinez OR, du Roscoat SR, Orgéas L (2016) Reversible dilatancy in entangled single-wire materials. Nat Mater 15:72-78
[3] Gadot B, Martinez OR, du Roscoat SR, Bouvard D, Rodney D, Orgéas L (2015) Entangled single-wire NiTi material: a porous metal with tunable superelastic and shape memory properties. Acta Mater 96:311-323

[4] Zarzour M, Vance J (2000) Experimental evaluation of a metal mesh bearing damper. J Eng Gas Turbines Power 122(2):326-329

[5] Ertas BH, Luo H (2008) Nonlinear dynamic characterization of oil-free wire mesh dampers. J Eng Gas Turbines Power 130(3):032503-1-8

[6] Liu P, He G, Wu LH (2008) Fabrication of sintered steel wire mesh and its compressive properties. Mater Sci Eng, A 489(1):21-28

[7] Zhang D, Scarpa F, Ma Y, Hong J, Mahadik Y (2014) Dynamic mechanical behavior of nickel-based superalloy metal rubber. Mater Des 56:69-77

[8] Ma Y, Zhang Q, Zhang D, Scarpa F, Liu B, Hong J (2014) A novel smart rotor support with shape memory alloy metal rubber for high temperatures and variable amplitude vibrations. Smart Mater Struct 23(12):125016

[9] Ma Y, Zhang Q, Zhang D, Scarpa F, Liu B, Hong J (2015) The mechanics of shape memory alloy metal rubber. Acta Mater 96:89-100

[10] Ma Y, Wang H, Li H, Hong J (2008) Study on metal rubber material's characteristics of damping and sound absorption. In: Proceedings of ASME Turbo Expo 2008, Berlin. GT2008-50961, pp 477-486

[11] Lee YB, Kim CH, Kim TH, Kim TY (2012) Effects of mesh density on static load performance of metal mesh gas foil bearings. J Eng Gas Turbines Power 134(1):012502

[12] San Andrés L, Chirathadam TA, Kim TH (2010) Measurement of structural stiffness and damping coefficients in a metal mesh foil bearing. J Eng Gas Turbines Power 132(3):032503

[13] Rannenberg M, Beer H (1980) Heat transfer by evaporation in capillary porous wire mesh structures. Lett Heat Mass Transf 7(6):425-436

[14] Ma Y, Zhang Q, Zhang D, Scarpa F, Liu B, Hong J (2015) Tuning the vibration of a rotor with shape memory alloy metal rubber supports. J Sound Vib 351:1-6

[15] Jiang G, He G (2014) Enhancement of the porous titanium with entangled wire structure for load-bearing biomedical applications. Mater Des 56:241-244

[16] Zhang D, Scarpa F, Ma Y, Boba K, Hong J, Lu H (2013) Compression mechanics of nickel-based superalloy metal rubber. Mater Sci Eng, A 580:305-312

[17] Li Y, Huang X, Mao W (2005) A theoretical model and experimental investigation of a nonlinear constitutive equation for elastic porous metal rubbers. Mech Compos Mater 41(4):303-312 
[18] Ma Y, Guo B, Zhu Z (2004) Static characteristics of metal rubber. J Aerosp Power 19(3):326-331 (In Chinese)

[19] Tan Q, Liu P, Du C, Wu L, He G (2009) Mechanical behaviors of quasi-ordered entangled aluminum alloy wire material. Mater Sci Eng A 527:38-44

[20] Liu P, Tan Q, Wu L, He G (2010) Compressive and pseudoelastic hysteresis behavior of entangled titanium wire materials. Mater Sci Eng, A 527(15):3301-3309

[21] Gibson LJ, Ashby MF (1997) Cellular Solids: structure and property, 2nd edn. Cambridge University Press, Cambridge

[22] Bažant ZP, Zhou Y, Daniel IM, Caner FC, Yu Q (2006) Size effect on strength of laminate-foam sandwich plates. J Eng Mater Technol 128(3):366-374

[23] Bazant ZP, Daniel IM, Li Z (1996) Size effect and fracture characteristics of composite laminates. J Eng Mater Technol 118(3):317-324

[24] Bažant ZP, Zhou Y, Novák D, Daniel IM (2004) Size effect on flexural strength of fiber-composite laminates. J Eng Mater Technol 126(1):29-37

[25] Ferro G, Carpinteri A (2008) Effect of specimen size on the dissipated energy density in compression. J Appl Mech 75(4):041003
[26] Wang H, Rongong J A, Tomlinson G R, Hong J (2010) Nonlinear static and dynamic properties of metal rubber dampers. In: Proceedings of ISMA 2010, Leuven. No. 2010-0064, pp 1301-1316

[27] Piedboeuf MC, Gauvin R, Thomas M (1998) Damping behavior of shape memory alloys: strain amplitude, frequency and temperature effects. J Sound Vib 214(5):885-901

[28] Boba K (2011) Characterisation of auxetic PU open cell foams with shape memory effect. West Pomeranian University of Technology, Szczecin, Master Dissertation

[29] Zhu B (2012) Theoretical and experimental investigation on compressive mechanical and thermophysical properties of metal rubber. PhD Dissertation, School of Energy and Power Engineering, Beihang University, Beijing, pp 22-49 (in Chinese)

[30] Ma Y, Liu X, Hong J (2015) Prediction for springback ratio of metal rubber based on BP neural network. 12th Academic Forum for Graduate Students at Beihang University, Beijing (in Chinese)

[31] Zhu B, Ma Y, Zhang D, Hong J (2012) A constitutive model of metal rubber based on hysteresis property. Acta Phys Sin, p 78101-078101 